\title{
Bases para la ordenacion de la pesca en la cuenca del Alto Tormes (Avila).
}

\author{
C. Alonso Gonzalez y D. Garcia de Jalon Lastra \\ Carlos Alonso Gonzalez y Diego Garcia de Jalon Lastra. \\ Laboratorio de Zoologia. E.T.S.I. de Montes, Avda. Ramiro de Maeztu s/n., 28040 Madrid, España.
}

\section{RESUMEN}

Se exponen los resultados procesados de las campafias de muestreo realizadas en tres tramos de montafia (1400 m) de la cuenca alta del río Tormes, dos en el propio Tormes (To-1 y To-2) y un tercero en un afluente del mismo (GBa), que sirven de base para analizar las caracteristicas de las poblaciones piscicolas y de su evolucion espacio-temporal, y establecer las bases científicas para la gestion tecnica de la pesca.

La composición de las comunidades piscicolas se estructura según la zonación longitudinal de los rios (IIlies \& Botosaneanu, 1969), adaptada a la cuenca del Duero por Garcia de Jalon \& Gonzalez del Tanago (1983). Las poblaciones de trucha se han analizado con mayor detalle, detectandose los mayores tamafios poblacionales en la Garganta de Barbellido (12 g. $\mathrm{m}^{-2}$ ) mientras que las del Tormes son escasas (menores de $5 \mathrm{~g} . \mathrm{m}^{-2}$ ). Estos tamafios de poblacion se comparan con los de otros rios y cuencas de la Peninsula. Las tasas de crecimiento tienden a aumentar conforme se baja en altitud, con coeficientes metabolicos entre 7 y 8 en los dos tramos mas altos (To-1 y GBa) y 12 en el tramo mas bajo (To-2). La mortalidad, la produccion y la tasa de renovacion anual siguen la misma pauta de diferenciacion entre los tramos altos y el bajo, aspecto que condiciona la estrategia de mejora en ambos casos.

Como propuestas de mejora se han establecido una serie de directrices que proponen una actuación sobre el habitat en To-1 y sobre la comunidad en To-2, no pareciendo necesaria una intervención en GBa.

Palabras clave: Ordenacion piscicola, pesca, trucha, gestion, Tormes.

\section{ABSTRACT}

Fish surveys were conducted in three mountain reaches (1400m a.s.l.) in the upper river Tormes basin (Avila, Spain). Samples were taken from the river Tormes itself (To-1 and To-2) and from a tributaiy of river Torrnes (GBa). Actual characteristics of fish populations, dynamics and distribution were examined, with an aim to establishing a scientific basefor their management.

Fish communities in rivers change according to a longitudinal pattern (Illies \& Botosaneanu, 1969). Garcia de Jalón \& Gonzalez del Tanago (1983) adapted this general model of change to the river Duero basin. Brown trout populations have been analysed in detail, estimating largestpopulation sizes in Garganta de Barbellido $\left(12 \mathrm{~g} \mathrm{~m}^{-2}\right)$. Populations in river Tormes are scarce (less than $5 \mathrm{~g} \mathrm{~m}^{-2}$ ). Population sizes are compared with those obtained in other rivers and basins in Spain and Portugal. Growth rates estimated tend to increase at lower altitudes. Metabolic rates ranged between 7 and 8 in the highest reaches (To-1 and GBa), reaching 12 in the downstream reach (To-2). Mortality, annual production and annual renewal rate also increased downstream.

The strategyfor improvement of fisheries should take into account these changes. Measures are proposed to improvefisheries condition at reaches To-1 (to improve the habitat) and To-2 (to improve the fish community). Site GBa does not require intervention.

Keywords: Fisheries management, fishing, trout, Tormes.

\section{INTRODUCCIÓN}

La evolucion negativa de las poblaciones piscicolas, en particular las de trucha común, así como el aumento de la demanda de pesca recreativa son motivos que hacen necesaria una planificación seria del recurso pesquero. En especial en un área como la cuenca del alto Tormes donde la pesca deportiva representa, cada vez más, una de las mayores fuentes de ingresos para las poblaciones ribereñas.

La trucha comun (Salmo trutta Linnaeus, 1758) es la especie piscícola de más interés en la mayoria 
de los cursos altos de los rios de la Peninsula Iberica, y tal es el caso del rio Tormes en sus tramos mas altos. Asi, en el Diccionario GeográficoEstadístico-Histórico de mediados del siglo pasado se habla de El Barco de Avila destacando "...las esquisitas truchas del Tormes, que transportan a Madrid entre nieve ó escabechadas, habiendose sacado desde 7 y 8 libras que es 10 mas comun hasta de 13 y 15." (Madoz, 1849).

Para plantear un plan de gestion racional de la pesca es necesario conocer una serie de parámetros que caracterizan la dinamica de las poblaciones de peces (Gulland, 1978; Youngs \& Robson, 1978; Garcia de Jalon, 1984). No obstante conviene establecer primero una caracterizacion de las comunidades piscicolas existentes en el area de estudio, mediante el análisis de las especies de peces que las conforman y la proporción en que se encuentra cada una, determinando la especie o especies dominantes en cada tramo con el fin de establecer una zonación longitudinal y altitudinal de los cursos que componen la cuenca. Los parametros de mayor interes para la caracterizacion de una poblacion piscicola son: densidad, estructura de la poblacion, factores de condición, crecimiento, mortalidad, producción y tasa de renovación anual (Garcia de Jalon \& Schmidt, 1995).

De cualquier manera, una buena gestion de la pesca debe pasar por la mejora tanto del recurso pesca en si como del habitat que funciona como

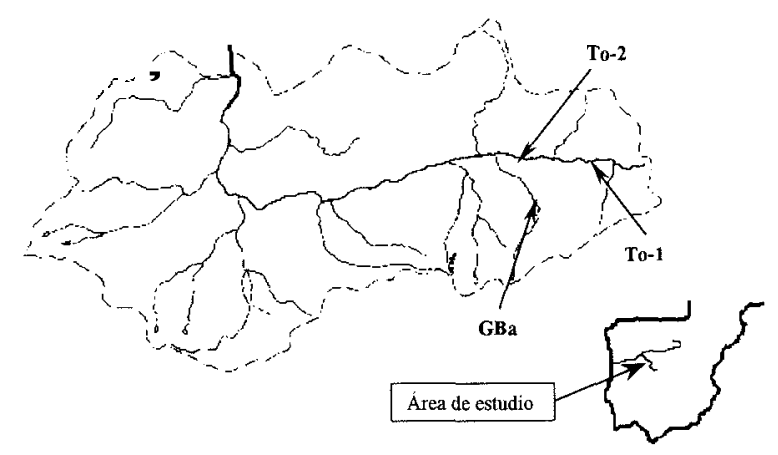

Fig. 1. Mapa esquematico de la red hidrográfica de la cuenca alta del rio Tormes y situación de las estaciones de muestreo dentro del area de estudio. Location of sampling stations in the upper river Tormes watershed (Avilu. Spain). soporte de dicho recurso. Es, por tanto, inútil establecer un plan de gestion de las poblaciones de trucha sin elaborar un plan de mantenimiento o mejora de las condiciones físicas y quimicas que caracterizan el habitat. Asi, dentro del estudio previo al establecimiento de las directrices de gestion se han de contemplar una serie de análisis del medio fisico y biologico, tales como: caracteristicas de la cuenca, riberas y orillas, regimen de caudales, granulometria, macrohabitats, caracteristicas físico-químicas de las aguas, vegetacion y usos y aprovechamientos de las zonas adyacentes al cauce y riberas.

\section{AREA DE ESTUDIO}

$\mathrm{El}$ area que ha servido de fuente para los datos del estudio que se expone comprende la subcuenca del rio Tormes desde su nacimiento hasta su union con la Garganta de Barbellido, en el sudoeste de la provincia de Avila al pie de la Sierra de Gredos, en el sector occidental del Sistema Central, en el limite con la provincia de Caceres.

En este territorio se han establecido tres estaciones en las que se han llevado a cabo cuatro muestreos en noviembre de 1997, julio de 1998, marzo de 1999 y julio de 1999. Parte de esta area se encuentra dentro de los limites de la Reserva Nacional de Caza de la Sierra de Gredos. En la figura 1 se ha representado la cuenca del rio Tormes en la provincia de Avila y la localización de las tres estaciones de muestreo.

Según la clasificacion fitoclimatica de Allue Andrade (1990), la zona de estudio estaria dentro de los climas IV(VI) mediterraneo subnemoral y $\mathrm{X}(\mathrm{IX})$ oroborealoide articoide termoxerico.

El rio Tormes en el area de estudio presenta un regimen de caudales claramente marcado por un maximo primaveral de $3.4 \mathrm{~m}^{3} / \mathrm{s}$ y un minimo estival no demasiado severo de $0.3 \mathrm{~m}^{3} / \mathrm{s}$ (datos procedentes de la estacion de aforos $\mathrm{n}^{\circ} 6$ de la Comisaria de Aguas del Duero, en el rio Tormes, T. M. de Hoyos del Espino).

Las estaciones de muestreo seleccionadas para el estudio son tres y sus principales características morfologicas son: 


\begin{tabular}{lcclll}
\hline \multirow{2}{*}{$\begin{array}{c}\text { Estacion Altitud Anchura Vegetación Pendiente RR/LL } \\
\text { (m) }\end{array}$} & $(\mathbf{m})$ & & $(\%)$ & \\
\hline To-1 & 1470 & 6.7 & Pinar & 5 & 2.13 \\
To-2 & 1330 & 14.6 & Pastizal & 1.4 & 2.33 \\
GBa & 1440 & 13.2 & Pastizal & 2.4 & 2.85 \\
\hline
\end{tabular}

\section{METODOLOGÍA}

La recopilacion de datos piscicolas se realizó mediante pesca electrica y aplicando el sistema de muestreo de Lury (1947) o metodo de las capturas sucesivas sin remplazamiento a esfuerzo constante. Para ello se llevaron a cabo cuatro campañas de muestreo entre noviembre de 1997 y julio de 1999 en la estacion To-1 y tres campaiias entre julio de 1998 y julio de 1999 en To-2 y GBa. En cada campaña y en cada estacion se acoto un tramo de 40-60 $\mathrm{m}$ mediante redes de agalla realizandose un mínimo de tres pasadas de pesca electrica.

Despues de cada pasada de pesca electrica se midieron y pesaron todos los peces capturados, con una precision de $1 \mathrm{~mm}$ y $0.1 \mathrm{~g}$ en cada medida, respectivamente. Asímismo se extrajeron escamas entre la línea lateral y la aleta dorsal al 20\% de las truchas, para su posterior lectura. Tras esta operación los peces eran devuel- tos al rio a unos $50 \mathrm{~m}$ aguas abajo del limite inferior del tramo.

Para estimar las existencias y el tamaño de las poblaciones se siguio el metodo de Maxima Verosimilitud Ponderada de Carle \& Strub (1978).

La determinación de la edad se hizo mediante la combinación del estudio de las graficas de frecuencia de capturas por clases de talla segun el metodo de Petersen (1896) y escalimetria (Steinmetz \& Müller, 1991; Hinning \& West, 2000).

Para la evaluacion de la condicion corporal de las truchas se utilizo el factor de condicion de Fulton (Anderson \& Neumann, 1996).

Para caracterizar el crecimiento de los individuos se ajustaron los valores de edad y longitud a una curva exponencial segun el modelo de Von Bertalanffy (1938).

La evaluacion de los parametros de la ecuacion de Von Bertalanffy se llevo a cabo mediante la realización de iteraciones con la formula de Von Bertalanffy, introduciendo el valor constante de la longitud del alevin al nacer y calculando, de esa forma el valor de $t_{\theta}$, que es el que se va a utilizar para el ajuste.

El valor de la tasa instantanea de mortalidad se determino tomando logaritmos en la expresion que establece la variación en el tiempo del numero de individuos de una cohorte (Ricker, 1977).

La producción en biomasa por unidad de tiempo se estimo segun la adaptación del metodo grá-

Table 1. Composición de la comunidad piscicola de cada tramo estudiado en las campaiias de muestreo en terminos de biomasa $\left(\mathrm{g} \cdot \mathrm{m}^{-2}\right)$. Lease $\mathrm{T}=$ trucha; $\mathrm{C}=$ cacho; $\mathrm{B}=$ boga. Biomass of the fish community $\left(\mathrm{g} \mathrm{m}^{-2}\right)$ at the three sampling stations in a survey of three mountain reaches of the upper river Tormes (Avila, Spain). $T=$ trout $C=$ Leuciscus; $B=$ Chondrostoma.

\begin{tabular}{|c|c|c|c|c|c|c|c|c|c|}
\hline \multirow[b]{3}{*}{ Campaña } & \multicolumn{9}{|c|}{ Estacion } \\
\hline & \multicolumn{3}{|c|}{ To- 1} & \multicolumn{3}{|c|}{ To-2 } & \multicolumn{3}{|c|}{$\mathrm{GBa}$} \\
\hline & $\mathrm{T}$ & $\mathrm{C}$ & B & $\mathrm{T}$ & $\mathrm{C}$ & B & $\mathrm{T}$ & $\mathrm{C}$ & B \\
\hline Nov'97 & 0.82 & 0 & 0 & & & & & & \\
\hline Jul'98 & 5.30 & 0 & 0 & 3.37 & 12.86 & 0.69 & 5.76 & 0 & 0 \\
\hline Mar'99 & 1.16 & 0 & 0 & 1.63 & 22.59 & 4.01 & 2.77 & 0 & 0 \\
\hline Jul'99 & 3.41 & 0 & 0 & 4.56 & 18.95 & 1.69 & 28.32 & 0 & 0 \\
\hline Media & 2.68 & 0 & 0 & 3.18 & 18.13 & 5.46 & 12.29 & 0 & 0 \\
\hline Desviacion tipica & 2.10 & 0 & 0 & 1.47 & 4.92 & 1.70 & 13.97 & 0 & 0 \\
\hline
\end{tabular}


Table 2. Composición de la comunidad piscicola de cada tramo estudiado en las campafias de muestreo en términos de densidad (individuos. $\mathrm{m}^{-2}$ ). Density of fish species (ind $\mathrm{m}^{-2}$ ) in surveys of the three reaches sampled. $T=$ trout; $C=$ Leuciscus; $B=$ Chondrostoma.

\begin{tabular}{|c|c|c|c|c|c|c|c|c|c|}
\hline \multirow[b]{3}{*}{ Campaña } & \multicolumn{9}{|c|}{ Estacion } \\
\hline & \multicolumn{3}{|c|}{ To-1 } & \multicolumn{3}{|c|}{ To-2 } & \multicolumn{3}{|c|}{$\mathrm{GBa}$} \\
\hline & $\mathrm{T}$ & $\mathrm{C}$ & $\mathrm{B}$ & $\mathrm{T}$ & $\mathrm{C}$ & B & $\mathrm{T}$ & $\mathrm{C}$ & B \\
\hline Nov'97 & 0.086 & 0 & 0 & & & & & & \\
\hline Jul'98 & 0.264 & 0 & 0 & 0.096 & 0.884 & 0.071 & 0.136 & 0 & 0 \\
\hline Mar'99 & 0.080 & 0 & 0 & 0.062 & 1.889 & 0.187 & 0.125 & 0 & 0 \\
\hline Jul'99 & 0.174 & 0 & 0 & 0.166 & 1.624 & 1.640 & 2.370 & 0 & 0 \\
\hline Media & 0.151 & 0 & 0 & 0.108 & 1.466 & 1.633 & 0.877 & 0 & 0 \\
\hline Desviacion tipica & 0.087 & 0 & 0 & 0.053 & 0.521 & 0.874 & 1.293 & 0 & 0 \\
\hline
\end{tabular}

fico de Allen al modelo de crecimiento de Von Bertalanffy (Allen, 1971). Las hipotesis que se siguieron son la de crecimiento isometrico y la de que para $t=0$ el peso $W=0$.

La tasa de renovacion anual se obtuvo a través del cociente producción/biomasa.

\section{RESULTADOS}

\section{Comunidades piscicolas}

Las especies detectadas en el area de estudio mediante las campaiias de pesca electrica en las

To-1

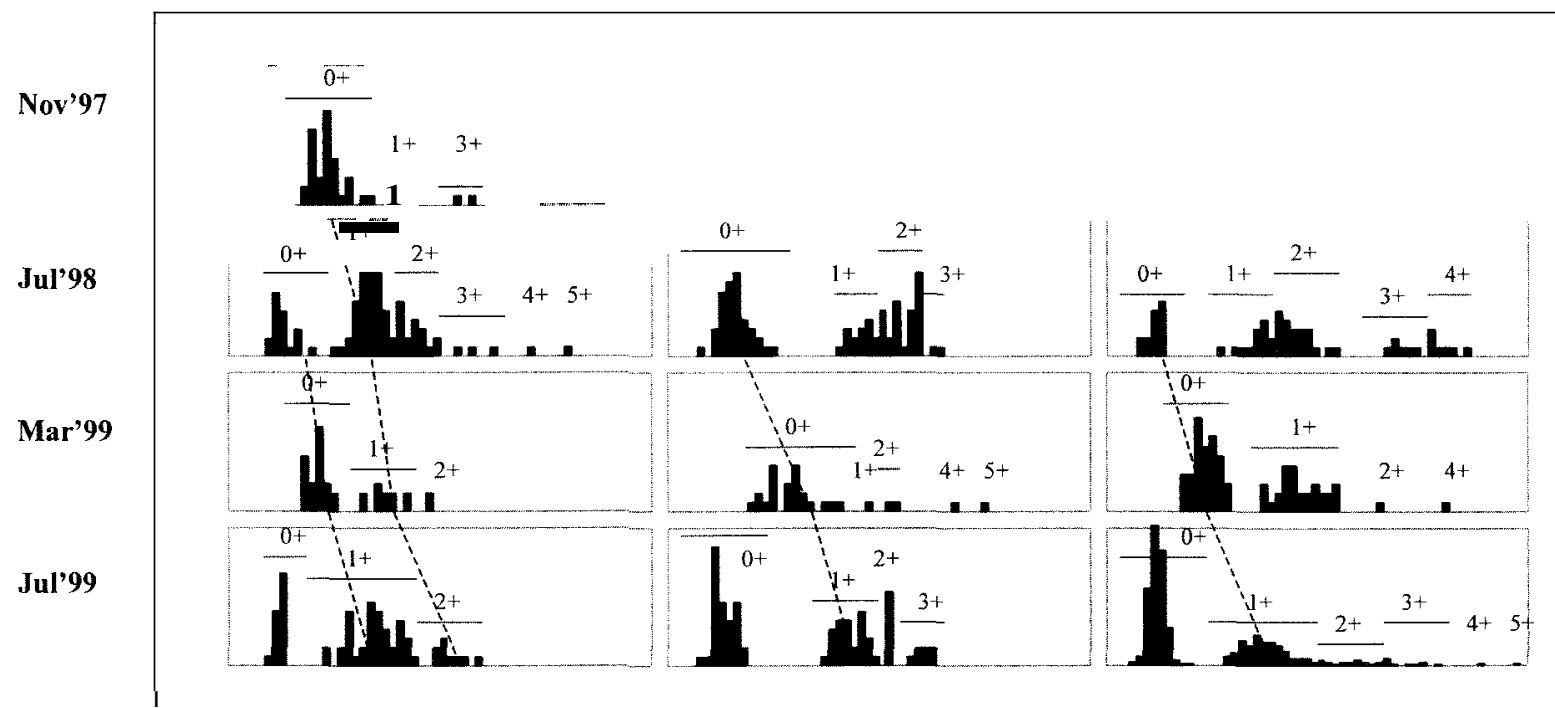

Fig. 2. Distribución de frecuencias de captura por clases de talla y asignacion de edad para las tres estaciones de muestreo en cada campaña. Las líneas intergraficas señalan la evolución de las cohortes identificadas. Capturefrequencies of each size class and age grooup in the three reaches sampled of the upper river Tormes watershed (Avila, Spain). Dotted lines indicate the identified cohorts. 
estaciones de muestreo antes referidas son tres: trucha comun (Salmo trutta L., 1758), cacho (Leuciscus curolitertii Doadrio, 1987) y boga (Chondrostomapolylepis Steindachner, 1865).

En las Tablas 1 y 2 se han recogido los valores de biomasa y densidad, respectivamente, detectados en cada campaiia para cada una de las estaciones muestreadas. En ellas se puede apreciar tanto la distribucion por especies de la comunidad de cada tramo como el tamaiio de las poblaciones de cada especie.

\section{Estructura de las poblaciones por tallas y edades}

Mediante el análisis de la distribucion de frecuencias de capturas por clases de talla y la lectura de escamas de los individuos de edad se ha podido determinar la edad de los individuos capturados y estimar unas fechas límite para la eclosion de los huevos. Esta parece tener lugar a finales de abril en los tres tramos estudiados. De la anterior asignacion de edades se han establecido los limites de talla entre los que se encuentra cada clase de edad en cada estacion y campaiia de muestreo, valores que se han recogido en la Tabla 3 .
En la figura 2 se han representado las distribuciones de frecuencias por tallas de las truchas capturadas en las sucesivas campaiias llevadas a cabo en las tres estaciones de muestreo. En ellas se han seiialado las evoluciones de las cohortes nacidas en los aiios 1997 y 1998 en la estacion To-1 y de las nacidas en 1998 en las estaciones To-2 y GBa.

En la estacion To-1 se puede apreciar una distribucion bimodal en las cohortes nacidas en 1997 y 1998 que se atenua con el tiempo en la primera y se mantiene en la segunda. La garganta de Barbellido, por el contrario, mantiene una distribucion unimodal para la cohorte de 1998 durante las tres campañas. La cohorte de 1998 en To-2 pasa de una distribucion unimodal en 1998 a una bimodal en 1999.

La evolución de la estructura de la poblacion por clases de edad en terminos de densidad a 10 largo del periodo de tiempo en el que se llevaron a cabo las campaiias de pesca eléctrica se ha representado en la figura 3 .

En las tres estaciones se puede observar un progresivo aumento de los efectivos, sobre todo de las clases de edad mas jovenes, entre las campañas de 1998 y 1999.

Table 3. Rango de longitudes entre las que se comprende cada clase de edad en cada estacion de muestreo y para todas las campaiias. Sizeclasses considered at different sampling stations in surveys conducted in three mountain reaches of the upper river Tormes (Avila, Spain).

\begin{tabular}{|c|c|c|c|c|c|c|c|}
\hline Fecha & Estacion & $0+$ & $1+$ & $2+$ & $3+$ & $4+$ & $5+$ \\
\hline \multirow{3}{*}{ Nov'97 } & To- 1 & $70-115$ & 130 & & $175-185$ & & \\
\hline & To-2 & & & & & & - \\
\hline & $\mathrm{GBa}$ & & & & & & - \\
\hline \multirow{3}{*}{ Jul'98 } & To-1 & $45-75$ & $90-130$ & $130-160$ & $175-200$ & 225 & 250 \\
\hline & To-2 & $40-90$ & $135-155$ & $160-180$ & 185 & & \\
\hline & $\mathrm{GBa}$ & $40-55$ & $95-130$ & $130-175$ & $210-230$ & $240-265$ & \\
\hline \multirow{3}{*}{ Mar'99 } & To- 1 & $70-90$ & $110-140$ & 155 & & & \\
\hline & To-2 & $75-135$ & 155 & $170-175$ & & 215 & 235 \\
\hline & $\mathrm{GBa}$ & $70-100$ & $125-175$ & 205 & & 250 & \\
\hline \multirow{3}{*}{ Jul'99 } & To-1 & $45-55$ & $85-145$ & $160-190$ & & & \\
\hline & To-2 & $40-70$ & $125-160$ & 170 & $185-200$ & & \\
\hline & $\mathrm{GBa}$ & $35-75$ & $100-140$ & $145-200$ & $205-245$ & 275 & 300 \\
\hline
\end{tabular}




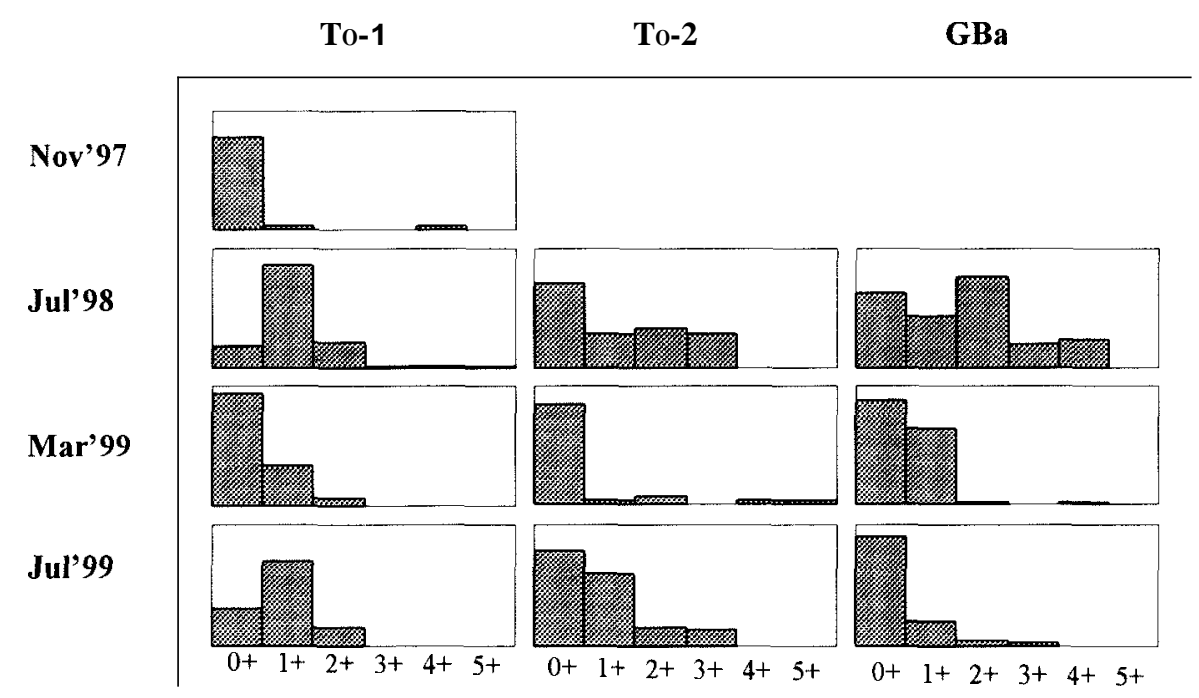

Fig. 3. Estructuras de la poblacion en cada estacion de muestreo para todas las campaiias de pesca eléctrica realizadas. Structure of populations at each sampling station, from electro-fishing campaigns.

\section{Factor de condicion}

En la tabla 4 se recogen los valores del factor de condicion de las poblaciones de trucha en cada estacion a lo largo de las campañas que se han llevado a cabo. De la observación de los valores medios del factor de condicion se puede apreciar una diferencia entre los valores de este coeficiente morfometrico en las truchas que habitan los dos tramos mas altos de los estudiados (To-I y GBa) y el mas bajo (To-2). Se encuentran, pues, truchas mas gruesas en la estacion mas baja de las tres estudiadas que en los dos tramos mas altos.

\section{Crecimiento}

En la figura 4 se han representado las curvas de crecimiento de Von Bertalanffy para las poblaciones de trucha comun de los tres tramos estudiados. Se ha diferenciado, tambien, con un codigo de tramas las series correspondientes al crecimiento de cada cohorte, de forma que se pueden apreciar las variaciones del crecimiento entre las cohortes nacidas en aiios distintos.
La ecuacion de crecimiento de Von Bertalanffy toma para las poblaciones de trucha de cada tramo estudiado la siguiente forma:

para To-1: $\mathrm{L}=34.67\left(1-e^{-0.2059(t+0.4396)}\right)$ con $\mathrm{r}^{2}=$ 0.9557 ,

para To-2: $\mathrm{L}=24.62\left(1-\mathrm{e}^{-0.5054(\mathrm{t}+0.2571)}\right) \operatorname{con} \mathrm{r}^{2}=$ 0.9534

y para GBa: $\mathrm{L}=45.78\left(1-\mathrm{e}^{-0.1747(\mathrm{t}+0.3880)}\right) \operatorname{con} \mathrm{r}^{2}=$ 0.9707 . Siendo $\mathrm{r}^{2}$ el valor del coeficiente de regresion del ajuste realizado a los valores reales de edad y longitud para obtener la ecuacion.

El coeficiente metabolico k.L $\mathrm{L}_{\infty}$, asi como el crecimiento en de las truchas en centímetros durante el primer año de vida, tiene un valor mas intuitivo para comparar los crecimientos de las poblaciones de cada estacion. Los valores que toman estos parametros para los tres tramos estudiados son:

para To-1: $\mathrm{k} . \mathrm{L}_{\infty}=7.14$ y $6.26 \mathrm{~cm}$ año ${ }^{-1}$, para To-2: $\mathrm{k} . \mathrm{L}_{\infty}^{\infty}=12.44$ y $7.81 \mathrm{~cm}$ año ${ }^{-1}$ y para GBa: $k . L_{\infty}^{\infty}=8.00$ y $7.13 \mathrm{~cm}$ año ${ }^{-1}$.

Considerando los anteriores valores se observa una notable diferencia entre los coeficientes metabolicos de los dos tramos mas altos (To-1 y GBa) y el tramo mas bajo de los estudiados (To- 
Table 4. Valores medios del factor de condicion de Fulton $(\mathrm{K})$ por estacion observados en cada campafia de muestreo. Mean values of the Fulton conditionfactor $(K)$ for each sampling station in surveys surveys conducted in three mountain reaches ofthe upper river Tormes (Avila, Spain).

\begin{tabular}{lccccc}
\hline Estacion & Nov'97 & Jul'98 & Mar'99 & Jul'99 & Media \\
\hline To-1 & 1.027 & 1.111 & 1.252 & 1.150 & 1.135 \\
To-2 & & 1.209 & 1.228 & 1.238 & 1.225 \\
GBa & 1.128 & 1.154 & 1.080 & 1.121 & 0.093 \\
\hline
\end{tabular}

Table 5. Produccion en g m-2año-1 (P) y tasa de renovacion anual en afio-I (P/B) de las poblaciones de trucha de cada tramo. Annual production $(P$; in $g$ m-2year- 1$)$ and annual renewal rate $(P / B$; in year-1) of trout populations in reaches sampled in three mountain reaches of the upper river Tormes (Avila, Spain).

\begin{tabular}{lcccccrrr}
\hline & $0+$ & $1+$ & $2+$ & $3+$ & $4+$ & $5+$ & Total \\
\hline Estacion & $\mathrm{P}$ & $\mathrm{P}$ & $\mathrm{P}$ & $\mathrm{P}$ & $\mathrm{P}$ & $\mathrm{P}$ & $\mathrm{P}$ \\
\hline To-1 & 0.38 & 0.73 & 0.15 & 0.01 & 0.01 & 0.002 & 1.28 \\
To-2 & 2.24 & 1.26 & 0.38 & 0.39 & 0.02 & 0.61 & 0.31 \\
GBa & 9.17 & 2.44 & 0.69 & 0.35 & 0.09 & 0.01 & 12.77 \\
\hline
\end{tabular}

2) teniendo las truchas de este ultimo tramo un coeficiente metabolico que supera en un $60 \%$ al de las truchas de los tramos mas altos.

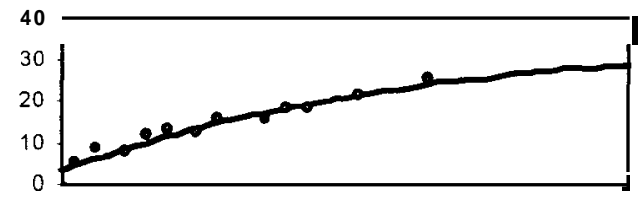

To-2

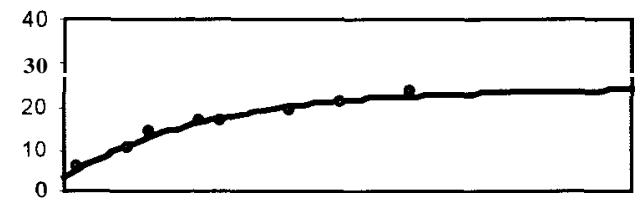

GBa

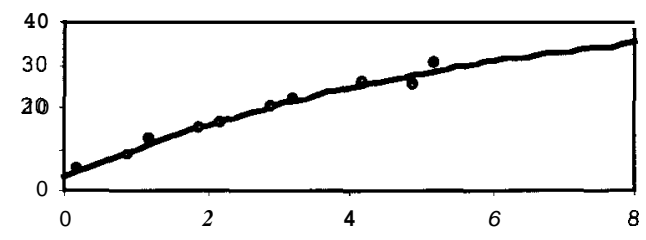

Fig. 4. Curvas de crecimiento de Von Bertalanffy para las poblaciones de trucha de cada tramo estudiado, representada la edad en el eje de abscisas y la longitud en el eje de ordenadas. Von Bertalanffy growth curves of brown troutpopulations in sampled reaches ofthe upper river Tormes watershed (Avila, Spain). Age is on the horizontal axis and length on the vertical axis.

\section{Mortalidad}

La tasa de mortalidad $\mathrm{Z}$ toma un valor relativamente alto en la estacion To-1 ( $\mathrm{Z}=1.0398)$ obtenida a partir de un ajuste de los datos de numero de individuos y edades con un coeficiente de regresion $\mathrm{R}^{2}=0.8619$, el valor de $\mathrm{Z}$ queda atenuado en To-2 respecto a la estacion anterior $(Z=0.7462)$ con un peor ajuste de los datos $\left(\mathrm{r}^{2}=0.7462\right)$; en la estacion de la Garganta de Barbellido (GBa) la mortalidad es la mas alta de las tres $(Z=1.1409)$ y el ajuste similar a To-1 $\left(r^{2}=0.8429\right)$. En definitiva, y de forma analoga a los casos de composición de la comunidad piscicola, factor de condicion y crecimiento se puede establecer una diferenciacion entre los dos tramos mas altos (To-1 y GBa) y el más bajo de los muestreados (To-2), presentado la poblacion de este ultimo una mortalidad mas baja que los anteriores.

\section{Produccion}

Los valores de la producción anual por clases de edad de las poblaciones de trucha comun en los tramos estudiados quedan recogidos en la Tabla 5. En ella se puede apreciar una notable diferen- 
cia en el valor de la produccion, tanto la total como la de las clases de edad pescables, entre la poblacion de la Garganta de Barbellido y las de los tramos del rio Tormes. Entre los dos tramos del Tormes tambien se aprecia diferencia entre uno y otro, siendo considerablemente mayor la produccion anual de la poblacion del tramo mas bajo de los dos (To-2).

\section{Tasa de renovacion anual}

En la tabla 5 se han recogido los valores de la tasa de renovacion anual de los tres tramos muestreados. Se puede apreciar la misma pauta de diferenciacion entre los tramos mas altos (To-1 y GBa) y el mas bajo (To-2) que en los parametros analizados en los apartados anteriores. Siendo en este caso el coeficiente Producción/Biomasa mayor en To-2 que en las otras dos estaciones.

\section{DISCUSIÓN}

La evaluación de los parametros poblacionales expuestos a 10 largo de este estudio, asi como el análisis de los factores bioticos y abioticos que tienen alguna influencia en el estado de las poblaciones de trucha comun de la cuenca del rio Tormes en Avila, permite sacar una serie de conclusiones que pueden orientar la gestion del medio fluvial para establecer un correcto aprovechamiento del recurso pesquero en dicho area.

La trucha común es la unica especie que esta presente en todas las estaciones de muestreo y constituye la especie dominante en las estaciones de la Garganta de Barbellido y del vedado de Navarredonda. El regimen de caudales variable y las bajas temperaturas que alcanza el agua en el invierno parecen condicionantes para la existencia de este tipo de comunidades monoespecificas de trucha comun. Así pues se puede incluir este tramo dentro del Epirhithron segun la clasificación de Garcia de Jalon \& Gonzalez del Tánago (1983).

A medida que el Tormes va bajando de cota se van incorporando especies propias del curso medio del rio, tales como el cacho y la boga, pre- sentes a partir de la estacion To-2, pudiendose clasificar la comunidad como perteneciente al Metarhithron, con presencia de trucha pero con abundancia de cachos y bogas. Analizando la evolución en el tiempo de esta comunidad 10 mas destacable es el notable aumento de la representacion de las bogas en Julio de 1999, teniendo en cuenta el caracter migrador y gregario de este pez se puede justificar este aumento de la poblacion de bogas como consecuencia de las reproducciones en Marzo de un gran numero de adultos inmigrantes de aguas abajo.

En terminos generales, de los tres tramos estudiados, los dos del rio Tormes albergan poblaciones de trucha comun que pueden considerarse escasas, tanto comparandolas con trabajos similares realizados en otros rios y cuencas como al contrastarlas con los rangos establecidos por Garcia de Jalon \& Schmidt (1995), segun los cuales, y por 10 general, valores de biomasa inferiores a 5 g.m $\mathrm{m}^{-2}$ pueden considerarse escasos.

Por otro lado, la poblacion de la Garganta de Barbellido presenta un valor de biomasa bastante alto que, teniendo en cuenta el caracter acido de sus aguas, menos productivas que las calizas (Frost \& Brown, 1971), puede llevar a considerarla como abundante.

El aumento tan notable de la poblacion experimentado entre Julio de 1998 y Julio de 1999 puede ser debido a la posible inmigracion de truchas de otros tramos de la garganta referida anteriormente.

Esta valoracion se refuerza al comparar los valores de biomasa de las poblaciones estudiadas con los registrados en otras cuencas y rios de la Peninsula Ibérica. La Garganta de Barbellido presenta un valor de biomasa que hace figurar a su poblacion truchera entre las de tamaño medio de las estudiadas por el Laboratorio de Hidrobiologia de la E.T.S.I. de Montes de la Universidad Politecnica de Madrid para diferentes puntos de la Peninsula Iberica (Datos no publicados). No obstante, que sus aguas sean silíceas $\mathbf{y}$ menos productivas que las calizas, permitiria calificar a su poblacion como abundante. Las biomasas medias detectadas en otros rios silíceos, y en el mismo rio Tormes entre Noviembre de 
Table 6. Valores de la biomasa $\left(\mathrm{g} \cdot \mathrm{m}^{-2}\right)$, produccion anual $\left(\mathrm{g} \cdot \mathrm{m}^{-2} \cdot \mathrm{año}^{-1}\right)$ y tasa de renovacion anual $\left(\mathrm{año}^{-1}\right)$ de las poblaciones de trucha comun estudiadas en otras cuencas. Biomass $\left(\mathrm{g} \mathrm{m}^{-2}\right)$, annual production $\left(\mathrm{g} \mathrm{m}^{-2}\right.$ year $\left.\mathrm{r}^{-1}\right)$ and annual renewal rate (year.') of brown troutpopulations in other basins.

\begin{tabular}{|c|c|c|c|c|c|}
\hline Rio & Cuenca/País & $\mathrm{B}$ & $\mathrm{P}$ & PIB & Referencia \\
\hline Arnoia & Miiio & 8.2 & 2.7 & 0.3 & GARCIAA DE JALON et al. (1990) \\
\hline Ulla & Norte & 1.3 & 2.9 & 2.2 & GARCIA DE JALON et al. (1990) \\
\hline Sor & Norte & 3.0 & 3.6 & 1.2 & GARCÍA DE JALON et al. (1990) \\
\hline Lor & Miiio & 2.9 & 9.3 & 3.2 & GARCÍA DE JALON et al. (1990) \\
\hline Eo & Norte & 6.0 & 7.6 & 1.3 & GARCÍA DE JALON et al. (1990) \\
\hline Ucero & Duero & 45.3 & & & LOBON-CERVIA et al. (1985) \\
\hline Orbigo & Duero & 47.5 & & & LOBON-CERVIA et al. (1985) \\
\hline Tormes & Duero & 5.4 & & & $\begin{array}{l}\text { MARTÍNEZ SÁNCHEZ-PALENCIA \& } \\
\text { GARCÍA DE JALON (1988) }\end{array}$ \\
\hline Shelligan Brook & Escocia & 6.4 a 10.2 & 7.7 a 12.3 & 1.2 & EGGLISHAW (1970) \\
\hline Walla Brook & Reino Unido & 6.7 a 12.0 & 10.0 a 18.0 & 1.5 & HORTON (1961) \\
\hline Bere Stream & Reino Unido & 1.7 a 8.7 & 2.6 a 13 & 1.5 & MANN (1971) \\
\hline Tarrant Brook & Reino Unido & 4 & 12 & 3 & MANN (1971) \\
\hline Devil's Brook & Reino Unido & 2.4 & 4.8 & 2 & MANN (1971) \\
\hline Docken's Stream & Reino Unido & 6.3 & 12 & 1.9 & MANN (1971) \\
\hline
\end{tabular}

1985 y Octubre de 1986, quedan recogidas en la Tabla 6, asi como los valores de produccion y tasa de renovacion anual.

El análisis del estado y la biomasa de las comunidades de macroinvertebrados de cada una de las estaciones (Datos no publicados) indica que la relación entre las biomasas de bentos y de peces tiene valores menores de 1 para todos los tramos estudiados siendo especialmente bajo en la estacion To-2 (0.006) y con valores parecidos entre si las otras dos $(0.034$ y 0.029 en To- 1 y $\mathrm{GBa}$, respectivamente). Esto indica que la disponibilidad de alimento puede constituir uno de los factores limitantes del tamaiio de las poblaciones del area de estudio (Garcia de Jalon et al., 1988).

Las poblaciones de trucha comun de la cuenca alta del Tormes estan, en general, bien estructuradas. Si bien se aprecian los efectos de las grandes crecidas registradas en Marzo de 1998, presentando una escasez de efectivos en la clase de edad 0+ durante los muestreos de Julio de 1998 y habiendo provocado una desestructuracion local de las poblaciones en ese año. No obstante es destacable la recuperación que presenta la estructura de las poblaciones observada en el muestreo de Julio de 1999, que hace que se consideren las condiciones del rio durante ese aiio como las ade- cuadas para el mantenimiento y desarrollo de unas poblaciones de trucha en esta parte de la cuenca. Por otro lado, parece probable la existencia de un posible "cuelo de botella" en las clases reproductoras en To-1 que seria la causa de la poca representación de la clase $0+$ en todas las campañas. Asi como una estructura poblacional rejuvenecida en $\mathrm{GBa}$, indicativa de que la garganta de Barbellido funcione como suministradora de alevines a toda la garganta y, probablemente, a los tramos altos del rio Tormes.

Las tasas de renovacion anual de los tres tramos son relativamente bajos, por 10 que se puede decir que dichos tramos albergan poblaciones de trucha en las que la probabilidad de que un individuo llegue a viejo es baja. Por tanto, en referencia a la selección de estrategias de la " $r$ " y de la "K" se puede afirmar que las truchas de estos tramos altos de la cuenca del Tormes utilizan la estrategia " $K$ ", 10 que implica que entre los factores que controlan sus poblaciones predominan aquellos relacionados con mecanismos densodependientes (competencia, depredación, parasitismo...), indicando que las caracteristicas del habitat no permiten a los tramos estudiados albergar poblaciones mucho mayores de trucha de las que actualmente existen. 
No obstante para valorar en detalle las tasas de renovacion observadas en el Tonnes, se ha estudiado la posición relativa de los puntos (produccion, biomasa) de cada estacion, respecto al estimador de relacion $\mathrm{P}=1.5 \mathrm{~B}$ propuesto por Chapman (1978) para aguas frias, y que coincide con el valor medio de las relaciones $\mathrm{P} / \mathrm{B}$ recogidas en la Tabla 6 para poblaciones de trucha en otros rios silíceos y/o de regimenes térmicos fríos.

\section{Bases para la ordenacion de la pesca}

Las estaciones To-I y GBa presentan valores bajos en la tasa de renovacion anual $\left(0.5 \mathrm{año}^{-1} \mathrm{y}\right.$ 1 año ${ }^{-1}$, respectivamente) $\mathrm{y}$, por tanto un cierto alejamiento de dicha tendencia, si bien en To- 1 la relacion $\mathrm{P} / \mathrm{B}$ es mucho menor que en $\mathrm{GBa}$, se puede ver que en ambas la produccion esta por debajo de lo que corresponderia a sus respectivas biomasas, según la citada tendencia de otras poblaciones. Esta circunstancia, unida al hecho de que en ambas estaciones la presion extractiva de la pesca es escasa o nula (To-1 es un vedado y GBa un coto de pesca sin muerte), parece indicar que las poblaciones de trucha estan cercanas al maximo que pueden albergar sus respectivos habitats. De forma que solo actuando sobre el habitat se podria favorecer el incremento de la biomasa de sus poblaciones de truchas.

Una poblacion en la que la tasa de renovacion anual fuese muy alta, es decir que la produccion quedase muy por encima de la relacion de referencia $\mathrm{P}=1.5 \mathrm{~B}$, seria mas susceptible de poder albergar una mayor biomasa $\mathrm{y}$, por tanto, podria resultar efectiva una actuación directa sobre el tamafio de la poblacion, por ejemplo favoreciendo el reclutamiento bien mediante la repoblacion con huevos embrionados o alevines procedentes de reproductores capturados en otros tramos del mismo rio, bien controlando poblaciones de peces que puedan competir con la trucha. No ocurre así en la estacion To-2, cuya tasa de renovacion anual alcanza un valor mas proximo a la media $\left(1.3 \mathrm{año}^{-1}\right), 10$ que puede hace mas dificil señalar las circunstancias sobre las que seria conveniente actuar.
Asi pues, y como bases para la ordenacion de los tramos estudiados se proponen las siguientes directrices:

En el caso de la primera estacion del Tormes en altitud (To-I) no parece muy probable que se pueda mejorar el tamaño y el estado de la poblacion de truchas. No obstante, cualquier propuesta de mejora deberia pasar por actuaciones sobre el habitat, intentando establecer un incremento en el numero de adultos que mejoren la reproducción y favoreciendo los factores que faciliten un aumento de la produccion de alimento (macroinvertebrados). Para ello seria conveniente la creación de pozas y zonas de refugio que puedan albergar buenos individuos reproductores. La construccion de rampas Hewitt modificadas de grandes piedras (Hewitt, 1934) puede resultar efectiva en este tipo de rios pequeños y de gradiente elevado.

En el segundo tramo del rio Tormes estudiado se podrian obtener resultados positivos a medio plazo en la poblacion de truchas si se actua directamente sobre la comunidad piscicola, habida cuenta del potencial de mejora que representa su tasa de renovacion anual, mayor que en los otros dos casos estudiados. De esta forma podria ser conveniente compensar la diferencia de presion de pesca entre las poblaciones de trucha y cacho, asi como controlar la contaminación quimica de las aguas mediante depuradoras, ya que las truchas son mas sensibles a la alteración fisica y química de las aguas que los cachos.

En cuanto a la estacion de la Garganta de Barbellido ( $\mathrm{GBa}$ ), no parece necesario ni conveniente intervenir, ni sobre el habitat ni sobre la comunidad. Si bien seria muy beneficioso para las poblaciones trucheras de una parte importante del area de estudio restaurar la conexión de los tramos altos de la garganta con el rio Tormes para permitir el acceso hasta las zonas de freza a los reproductores del Tormes o de los tramos bajos de la garganta. Asimismo seria conveniente la rehabilitación de las zonas de freza aguas abajo de la presa, mediante la revision de la concesion de caudal a la minicentral hidroeléctrica.

Para concluir y como orientaciones para la ordenacion para toda la cuenca alta, se propondria: 
La gestion integrada de toda la cuenca. Revisar la division actual de la cuenca en tramos libres, vedados y acotados, mediante el establecimiento de rotaciones. La creación de tramos libres sin muerte (Almodovar \& Nicola, 1998) o el aumento de la talla minima de pesca en los actuales tramos libres pueden funcionar como formas efectivas de actuacion sobre las poblaciones. Y, por ultimo, la recuperacion de las abundantes zonas de refugio de que disponian en los azudes de derivación y canales de riego y abastecimiento de molinos que actualmente se encuentran abandonados.

\section{BIBLIOGRAFÍA}

ALLEN, K. R. 1951. The computation of production in fish populations. N.Z. Sci. Rev., 8: 89.

ALLEN, K. R. 1971. Relation between production and biomass. J. Fish. Res. Bd. Canada, 28: 1573-1581.

ALLUE ANDRADE, J. L. 1990. Atlas fitoclimático de España. I.N.I.A., Madrid. 221 pp.

ALMODÓVAR, A. \& G. G. NICOLA. 1998. Assessment of a brown trout Salmo trutta population in the River Gallo (central Spain): angling effects and management implications (Salmonidae). Ital. J. Zool., 65, Suppl.: 539-543.

ANDERSON, R. O. \& R. M. NEUMANN. 1996. Length, Weight, and Associated Structural Indices. En: Fisheries Techniques. B.R. Murphy \& D.W. Willis (eds.). 732 pp. American Fisheries Society, Bethesda.

BAILEY, P. B. 1977. A method for finding the limits of application of the Von Bertalanffy growth model and statistical estimates of the parameters. J. Fish. Res. Bd. Can., 34: 1079-1084.

BEVERTON, R. J. H. 1954. Notes on the use of theoretical models in the study of the dynamics of exploited populations. U.S. Fish. Lab. Beaufort. N.C., Misc. Contrib., 2. 159 pp.

CARLE, F. L. \& M. R. STRUB. 1978. A new method for estimating population size from removal data. Biometrics, 34: 621-380.

CHAPMAN, D. W. 1978. Production in fish populations. En: Ecology of Freshwater Fish Production. S.D. Gerking (ed.). Blackwell Scientific Publications, Oxford. 520 pp.

COWX, I. G. 1983. Review of the methods for estimating fish population size from survey removal data. Fisheries Management, 14(2): 67-82.
EGGLISHAW, H. J. 1970. Production of salmon and trout in a stream in Scotland. J. Fish. Biol., 2: 117-136.

FORD, E. 1933. An account of the herring investigations conduced at Plymouth during the years from 1924-1933.J. Mar. Biol. Assoc., 19: 305-384.

FROST, W. E. \& M. E. BROWN. 1971.La trucha. Ed. Academia, S.L. Leon. 319 pp.

GARCÍA DE JALON, D. 1984. Los problemas de las truchas en los rios españoles. Quercus, 15: 10-12.

GARCÍA DE JALÓN, D. \& M. GONZÁLEZ DEL TÁNAGO. 1983. Estudio biotopologico de las comunidades piscicolas de la cuenca del Duero. Bol. Est. Centr Ecol., 12(24): 57-66.

GARCÍA DE JALÓN, D., C. MONTES, E. BARCELÓ, C. CASADO \& F. MENES. 1988. Effects of hydroelectric scheme on fluvial ecosystems within the Spanish Pynerees. Regulated Rivers: Research \& Management, 2: 479-491.

GARCÍA DE JALÓN, D., M. MAYO, F. HERVELLA, E. BARCELO \& C. VILLETA. 1990. Pesca fluvial en Galicia: Bases limnológicas para su gestion. Servicio Estudios e Publicacións. Secretaria Xeral Tecnica. Consellería de Agricultura, Ganderia e Montes. $145 \mathrm{pp}$.

GARCÍA DE JALÓN, D. \& G. SCHMIDT. 1995. Manual práctico para la gestion sostenible de la pesca fluvial. A.E.M.S. San Juan de las Abadesas (Gerona). $196 \mathrm{pp}$.

GULLAND, J. A. 1964. Manual of methods for fish population analysis. FAO. Fish. Tech. Pap., 40: 1-60.

GULLAND, J.A. 1978. Fishing, Fish and Food Production. En: Ecology of Freshwater Fish Production. S. D. Gerking (ed.): 387-402. Blackwell Sc. Pub. Oxford.

HEWITT, E. 1934. Hewitt's handbook of stream improvement. Marchbanks Press. N.Y. 82 pp.

HINING, K. J. \& J. L. WEST. 2000. Validation of scales and otholiths for estimating age of rainbow trout from southern Appalachian streams. North American Journal of Fisheries Management, 20: 978-985.

HORTON, P. A. 1961. The bionomics of brown trout in a Dartmoor stream. J. Anim. Ecol., 30. 311-338.

ILLIES, J. \& L. BOTOSANEANU. 1963. Problemes et methodes de la classification et de la zonation ecologique des eaux courantes considerees surtout du point de vue faunistique. Mitt. Int. Verein. Theor. Angew. Limnol., 12: 1-57.

LOBÓN-CERVIÁ, J., A. DE SOSTOA \& C. MONTAÑÉS. 1986. Fish production and its relation with the community structure in an aquifer-fed 
stream of Old Castile (Spain). Polskie Archiwum Hydrobiologii, 33: 333-343.

LURY, D. B. 1947. On the estimation of biological populations. Biometrics, 3(4): 145-167.

MADOZ, P. 1849. Diccionario GeográficoEstadístico-Histórico de España y sus Posesiones de Ultramar. Tomo IV, Madrid. P. Madoz (ed.).

MANN, R. H. K. 1971. The populations, growth and reproduction of fish in the River Thames. J. Anim. Ecol., 34: 155-190.

MARTÍNEZ SANCHEZ-PALENCIA, J. \& D. GARCÍA DE JALON. 1988. Estudio de las poblaciones trucheras del río Tormes. Ecología, 2: 303-314.

MORAN, P. A. P. 1951. A mathematical theory of animal trapping. Biometrika, 38. 307-311.

PETERSEN, C. G. J. 1896. The yearly inmigration of young plaice into Linsfjord from the German sea. Rep. Dan. Biol. Stu., 6: 1-48.
RICKER, W. E. 1977. The Historical Development. En: Fish Population Dynamis. J.A. Gulland, (ed.): 1-12. John Wiley \& Sons, Ltd., Chichester.

STEINMETZ, B. \& R. MÜLLER. 1991. An Atlas of Fish Scales and Other Bony Structures Used for Age Determination. Samara Publishing, Cardigan. 51 pp.

VON BERTALANFFY, L. 1938. A quantitative theory of organic growth. Hum. Biol., I0(2): 181-213.

WALFORD. L. A. 1946. A new graphic method of describing the growth of animals. Biol. Bull., 90(2): 141-147.

YOUNGS, W. D. \& D. S. ROBSON. 1978. Estimation of population number and mortality rate. Methods for Assessment of Fish Production in Fresh Waters. T. Bagenal (ed.): 137-164 Blackwell Sc. Pub. Oxford.

ZIPPIN, C. 1956. An evaluation of the removal method of estimating animal populations. Biometrics, 12: $163-189$. 